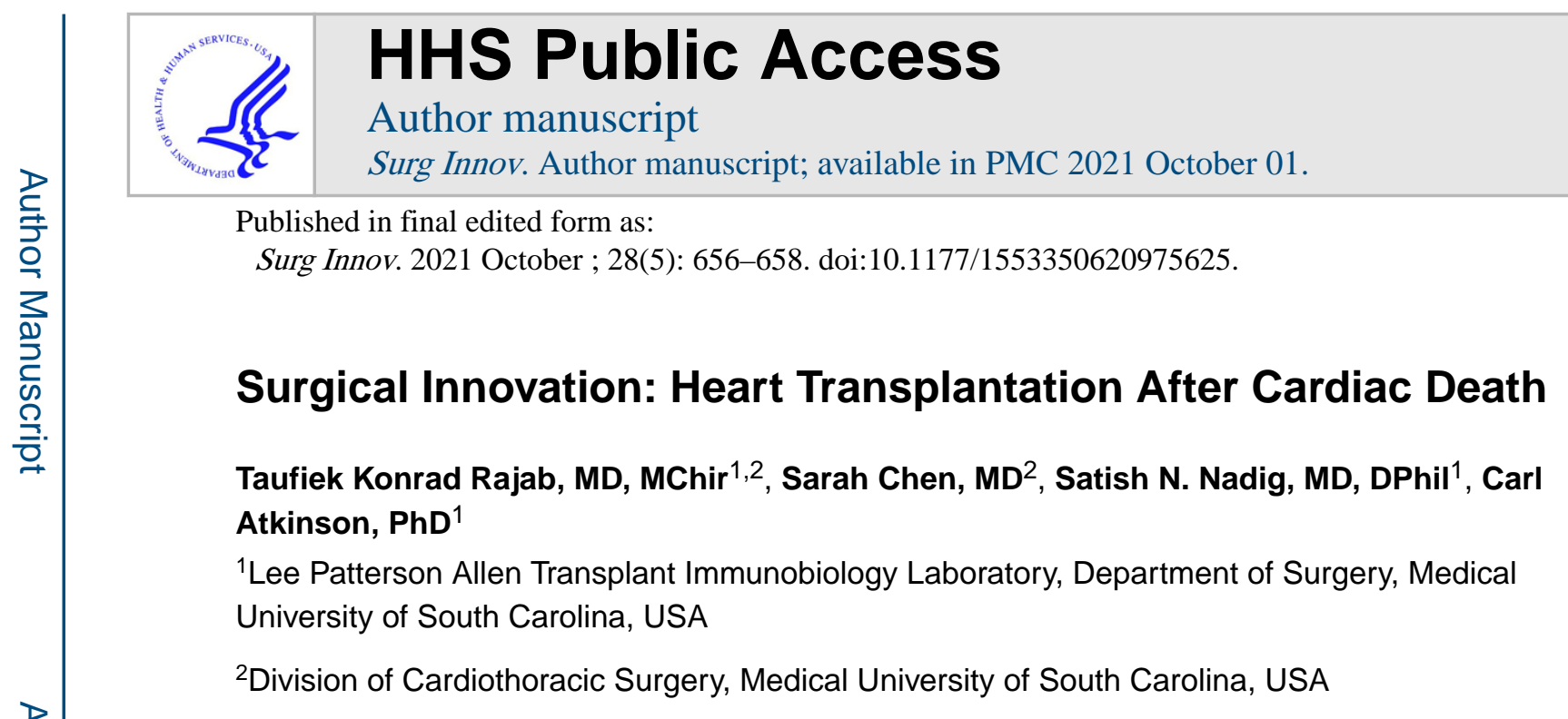

\title{
What is the Innovation?
}

The first clinical heart transplant was performed by Christiaan Barnard in South Africa in $1967 .{ }^{1}$ The donor was declared dead on the basis of cardiac arrest while the recipient awaited transplantation in an adjacent operating room. However, concerns about uncontrollable warm ischemic injury to the donor heart during cardiac arrest caused clinicians to abandon donation after cardiac death (DCD) heart transplantation once the legal and ethical frameworks for donation after brian death (DBD) became established. More recently, DCD heart transplantation was reexplored in response to the donor heart shortage. A breakthrough was achieved in Australia in 2014 when Kumud Dhital used a portable normothermic perfusion system to reanimate the DCD donor heart ex-vivo to perform the world's first distantly procured DCD heart transplant. ${ }^{2}$ Ex-vivo reanimation of the arrested donor heart allows for an evaluation of the warm ischemic injury sustained during cardiac death (Figure 1). However, critics in America argued that DCD invalidates the donor declaration of cardiac death, which is defined as irreversible cardiac arrest. In this scenario, DCD heart transplantation would violate the dead donor rule. ${ }^{3}$ Therefore, further development of the ethical and legal frameworks governing DCD heart transplantation was necessary before the first distantly procured DCD heart transplant could be performed in the United States in 2019. ${ }^{4}$

\section{What Are the Key Advantages Over Existing Approaches?}

The key advantage of DCD heart transplantation is the potential to increase the cardiac donor pool in the United States by more than $20 \%$ in the near future. ${ }^{5}$ This expands upon the concept of extended criteria donors and will lead to a decrease in wait-list mortality.

\footnotetext{
Article reuse guidelines: sagepub.com/journals-permissions

Corresponding Author: Taufiek Konrad Rajab, MUSC Shawn Jenkins Children's Hospital, 10 McClennan Banks Drive, Charleston 29425, SC, USA. t.rajab@gmail.com.

Author Contributions

Study concept and design: Taufiek Konrad Rajab, Satish N. Nadig, and Carl Atkinson

Acquisition of data: Taufiek Konrad Rajab and Sarah Chen

Analysis and interpretation: Taufiek Konrad Rajab and Sarah Chen

Study supervision: Taufiek Konrad Rajab, Satish N. Nadig, and Carl Atkinson

Declaration of Conflicting Interests

The author(s) declared no potential conflicts of interest with respect to the research, authorship, and/or publication of this article.
} 
A hypothetical advantage is that ischemic preconditioning during DCD heart procurement could protect the donor heart from ischemic injury during implantation.

\section{How Will This Affect Clinical Care?}

DCD heart transplantation will affect the clinical care of both organ donors and heart transplant recipients. On the organ donor side, the clinical protocols and surgical techniques that govern procurement will require adjustment if DCD hearts are procured. Importantly, priming of the normothermic perfusion system requires drainage of up to $2 \mathrm{~L}$ of donor blood before any organ protective perfusate can be given by the abdominal team. On the recipient side, the decisions for accepting a DCD heart and the process of informed consent will be affected by the limited clinical outcomes data for this new category of donors.

\section{Is There Evidence Supporting the Benefits of the Innovation?}

DCD heart transplantation is supported by clinical evidence from case series at avant-garde transplant centers in Europe and Australia. An analysis of 28 consecutive DCD heart transplants performed at Royal Papworth Hospital in Cambridge between 2013 and 2017 revealed a 1 -year mortality rate of $14 \%$, which did not differ significantly from matched DBD heart transplants. ${ }^{6}$ Similarly, an analysis of 23 consecutive DCD heart transplants performed at St Vincent's Hospital in Sydney between 2014 and 2018 revealed a mortality rate of $4.4 \%$ over a total follow-up period of 15500 days for the entire cohort. ${ }^{7}$ In the near future, further evidence evaluating DCD heart transplantation is expected from a prospective, randomized, and concurrent controlled clinical trial designed to compare the 6 months survival of 180 patients after either DCD or standard criteria DBD heart transplants. This trial with 25 activated centers in the United Sates is nearly fully enrolled (Clinicaltrials.gov Identifier NCT03831048).

\section{What Are the Barriers to Implementing This Innovation More Broadly?}

The barriers to implementing DCD heart transplantation more broadly are clinical, economical, and ethical. The clinical barriers to broader implementation are centered on the injury to the donor heart during cardiac death. Donor progression to cardiac death can add substantial warm ischemic time to the procurement, and uncontrolled ventricular fibrillation during cardiac death may damage the graft. Moreover, variations between hospital protocols for DCD standoff times add to the uncertainty. Other important barriers to implementing this innovation more broadly are formed by the contradictions inherent to DCD heart transplantation with the dead donor rule described above. This is an area of ethical and legal controversy where well-considered judgments differ widely, potentially leaving transplant teams vulnerable to criminal prosecution. ${ }^{3}$ These issues have given many surgeons a pause. Last but not least, cost represents an important barrier to wider implementation of DCD heart transplantation. The Transmedics Organ Care System heart ex-vivo perfusion console lists for US\$270 000 and each procurement requires consumables to house and perfuse the heart for US\$68 800. Transmedics also offers an optional program to provide clinical and surgical support on donor runs for an additional fee. In Europe, many DCD hearts are procured using the cheaper regional reperfusion protocol pioneered at Royal Papworth 
Hospital. This relies on in situ reanimation of the donor heart by what is effectively extracorporeal membrane oxygenation cardiopulmonary resuscitation administered to the donor. However, this protocol raises further ethical questions because it requires the procurement team to clamp the donor carotid arteries or risk inadvertently resuscitating the donor's brain. ${ }^{5}$ Therefore, this protocol is not used in the United States.

\section{In What Time Frame Will This Innovation Likely Be Applied Routinely?}

Based on the experience in Europe and Australia, DCD heart transplantation will likely be applied routinely by a moderate number of avant-garde transplant centers in the near future. However, the complexities of DCD heart procurement and the barriers described above mean that routine application of DCD heart transplantation by less aggressive centers will likely take some time.

\section{Acknowledgments}

Figure created with Biorender.com.

Funding

The author(s) received no financial support for the research, authorship, and/or publication of this article.

\section{References}

1. Barnard CN. A human cardiac transplant: An interim report of a successful operation performed at Groote Schuur hospital, Cape town. S Afr Med J. 1967;41:1271-1274. [PubMed: 4170370]

2. Dhital KK, Iyer A, Connellan M, et al.Adult heart transplantation with distant procurement and ex-vivo preservation of donor hearts after circulatory death: A case series. Lancet. 2015;385(9987):2585-2591. [PubMed: 25888085]

3. Rajab TK, Singh SK. Donation after cardiac death heart transplantation in America is clinically necessary and ethically ustified. Circ Heart Fail. 2018;11(3):e004884. [PubMed: 29664408]

4. Abbasi J“Donation after circulatory death" heart transplant is a US first. JAMA. 2020;323(2):111.

5. Rajab TK, Jaggers J, Campbell DN. Heart transplantation following donation after cardiac death: History, current techniques, and future. J Thorac Cardiovasc Surg. 2020; S0022-5223(20):30529.

6. Messer S, Page A, Axell R, et al.Outcome after heart transplantation from donation after circulatorydetermined death donors. J Heart Lung Transplant. 2017;36(12):1311-1318. [PubMed: 29173394]

7. Chew HC, Iyer A, Connellan M, et al.Outcomes of donation after circulatory death heart transplantation in Australia. J Am Coll Cardiol. 2019;73(12):1447-1459. [PubMed: 30922476] 


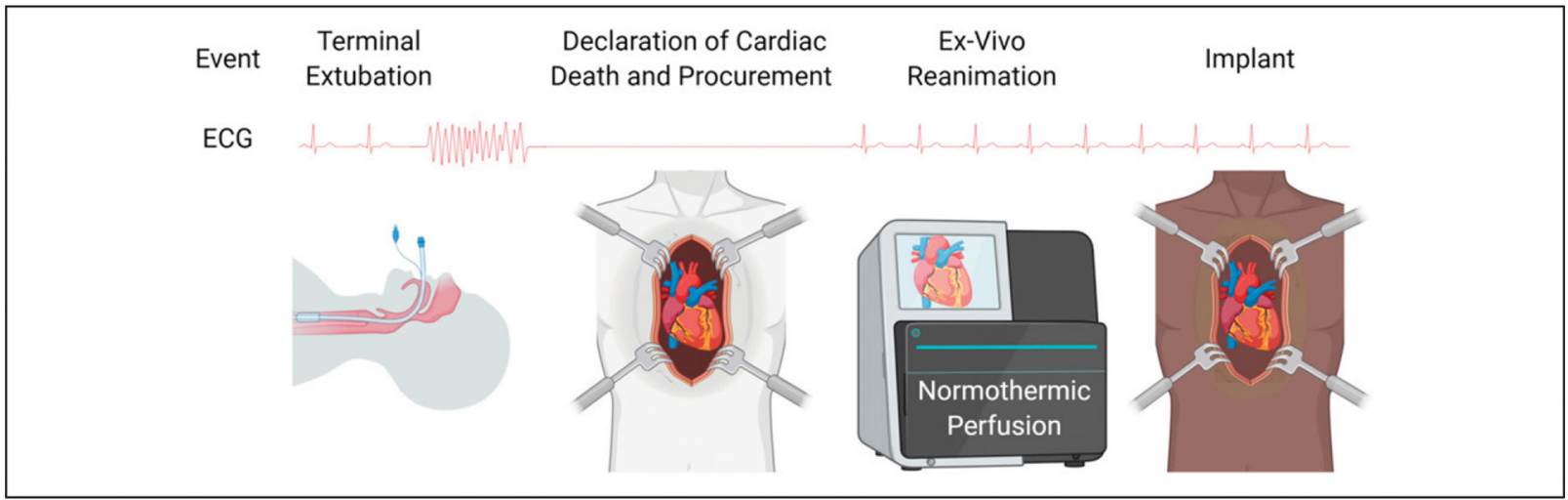

Figure 1.

Donation after cardiac death heart transplantation with ex-vivo reanimation. 